\title{
Johann Georg Zimmermann
}

1728-1795

Zu seinem 250. Geburtstag

Von Erwin H. Ackerknecht

Im Jahre 1928 widmete die Schweizer Medizinische Wochenschrift ihre ganze Nummer 49 dem Andenken jenes einst so berühmten, nun aber vergessenen Arztes aus Brugg. Die Nummer enthielt Beiträge von Bouvier, Sigerist, Karcher, Temkin und Kielholz Vater, also einer illustren Kompanie. Vielleicht entsprach diese gründliche Behandlung des Themas nicht einmal in erster Linie der Bedeutung des Dargestellten, sondern vor allem dem jugendlichen Enthusiasmus der erst sieben Jahre alten Schweizerischen Gesellschaft für Geschichte der Medizin und der Naturwissenschaften. Wie dem auch sei, Johann Georg Zimmermann ist in der Tat ein wesentlicher Vertreter einer der brilliantesten Perioden der schweizerischen Wissenschaftsgeschichte ${ }^{1}$ und war seinerzeit so berühmt, daß es sich doch wohl lohnt, ihn fünfzig Jahre später erneut zu betrachten.

Zimmermann, geboren in Brugg am 28. Dezember 1728 in eine lokale Patrizierfamilie $^{2}$, war von Kindheit an zweisprachig, da seine Mutter Französin war. 1747 entschloß sich der junge Mann, der infolge des frühen Todes seiner Eltern frei über sein Vermögen verfügte, Medizin zu studieren, und zwar in der berühmten neuen Universität Göttingen und bei dem ebenso berühmten Landsmann Albrecht Haller. Haller nahm ihn sehr freundlich auf - Zimmermann lebte in seinem Hause vier Jahre lang - und zeigte ihm ein Wohlwollen und eine Förderung, die Zimmermann ihm allerdings, nachdem er arriviert war, schlecht gelohnt hat. 1751 erschien Zimmermanns Dissertation «De irritatione», basiert auf 200 Tierexperimente, welche weitere Beweise zur Irritationslehre Hallers erbrachten. Zimmermann folgte Haller übrigens nicht nur auf diesem Gebiet, sondern auch z. B. in seiner in Göttingen naheliegenden Anglophilie. 1752 ließ sich Zimmermann mit Hallers Hilfe in Bern nieder und heiratete eine Verwandte desselben. 1754 wurde er, wiederum mit Hallers Hilfe, Stadtarzt in Brugg.

Hier entfaltete er, trotz einer sich dauernd ausweitenden Praxis, in 14 Jahren eine sehr fruchtbare literarische Tätigkeit. 1755 erschien sein Buch über Haller, 1756 die erste Fassung seines Buches über die Einsamkeit, 1763 die erste Fassung seines Buches über den Nationalstolz, 1763 die erste Fassung seines Buches über die medizinische Erfahrung, das übrigens unvollendet blieb - Bouvier hat einen Teil der überlebenden Manuskripte in seiner Monographie veröffentlicht. 1767 
veröffentlichte Zimmermann schließlich im Anschluß an einige lokale Epidemien sein Buch über Dysenterie.

Zimmermanns Briefe sind voll unaufhörlicher Klagen über seine Isolierung in der Kleinstadt und über seine «Hypochondrie». Esther Fischer-Homberger hat in ihrer Monographie ${ }^{3}$ dargelegt, daß der Ausdruck Hypochondrie um diese Zeit sich nicht mit unserer heutigen Bedeutung deckt, sondern eher dem entspricht, was wir heute als Neurose oder psychosomatisches Leiden bezeichnen. Zimmermanns fröhliches Verhalten auf den Sitzungen der seit 1761 im nahen Schinznach tagenden Neuen Helvetischen Gesellschaft spricht übrigens auch nicht für eine ausgesprochene Melancholie. Was ihn plagte, war wohl das Gefühl, nicht seiner Größe entsprechend plaziert zu sein. Haller und Tissot verschafften ihm schließlich die Nachfolge von Werlhof als Hofarzt in Hannover im Jahre 1768. Hier und im Bad Pyrmont führte er nun eine sehr glänzende, mondäne Existenz, die ganz und gar nicht dem entspricht, was er in seinem Buch über die Einsamkeit gepredigt hatte. Das einzige neue Werk, das er in Hannover schrieb, war das Buch über Friedrich den Großen (1790).

Persönlich trafen ihn eine Reihe Unglücksfälle: seine erste Frau starb 1770; 1771 mußte er sich in Berlin einer schmerzhaften Hernienoperation unterziehen; 1777 wurde sein Sohn unheilbar geisteskrank (auch Zimmermanns Mutter hatte so geendet) und ist bei dem Arzt Hotze in Richterswil jahrzehntelang verdämmert; 1781 starb seine Tochter an Lungenschwindsucht. Seine Praxis blühte, aber die Wendung des Hofrats vom republikanischen Aufklärer zum monarchistischen Konservativen, welche durch die Französische Revolution noch eine deutlich paranoide Färbung erhielt, zerstörte viele seiner Freundschaften. In den letzten Jahren seines Lebens war er eindeutig psychotisch und ist am 7.Oktober 1795 an einer Paranoia-bedingten Nahrungsenthaltung gestorben.

Als Mediziner begann Zimmermann als brillianter Haller-Schüler und -Mitarbeiter, aber er hat zum Bedauern seines Lehrers sich nicht auf dieser Linie weiterentwickelt. Sein Dysenterie-Buch ${ }^{4}$ ist von geringem Wert, trotz seines verdienstvollen Kampfes gegen abergläubische Dorfbarbiere, Henker, Harnschauer und dergleichen mehr. Ob seine abführende Behandlung wirklich weniger fatal war als die stopfende seiner Gegner, muß dahingestellt bleiben. Trotz solcher Schwächen gehört Zimmermann durchaus in das berühmte Schweizer medizinische Aufklärer- und Freundestrio Zimmermann, Tissot und J. C. Hirzel, welche die bis dato esoterische wissenschaftliche Medizin dem Volk verständlich machten und für die Präventivmedizin eine breite Bresche schlugen.

Zimmermanns bekanntestes medizinisches Buch «Von der Erfahrung in der Arzneykunst», welches sogar hundert Jahre später noch von Claude Bernard 
gelesen und zitiert wurde, ist im technisch-medizinischen Teil schwach, obwohl es wie alle seine Bücher interessante psychosomatische und psychologische Einsichten enthält. Das Wesentliche an diesem Buch ist der philosophische Teil. Zimmermann amüsiert sich zwar als Mediziner über die «Romane der Philosophen», aber auch er ist im wesentlichen Populärphilosoph und schreibt, wie schon Sigerist feststellte, eigentlich Literatur und nicht Wissenschaft. Das wird schon klar aus seinem flotten Erstling über Haller. Ersichtlich wird es auch aus seinem Freundeskreis. Zwar war er mit den Ärzten Hirzel und Tissot jahrzehntelang eng befreundet, aber mindestens so nahe stand ihm zum Beispiel der Theologe und Schwärmer Lavater, den er zu seiner Physiognomik inspiriert hatte, allerdings später als Mystiker angriff. Eng verbunden war er auch mit solchen Schriftstellern wie Bodmer, Wieland, dem in Berlin wirkenden edlen Pädagogen J.G.Sulzer (1720-1779), Herder, Goethe, Moses Mendelssohn und den Auchschriftstellern Katharina II. und Friedrich II.

Zimmermanns Buch über die Erfahrung bedient sich einer etwas billigen Dichotomie, welche er schon in seinen Büchern über den Nationalstolz (es gibt einen guten und einen schlechten Nationalstolz) und die Einsamkeit (es gibt eine gute und eine schlechte Einsamkeit) verwendet hatte. So gibt es bei ihm auch eine gute und eine "falsche», das heißt schlechte Erfahrung. Das Prinzip der «falschen Erfahrung» erweist sich aber gerade in seiner Anwendung auf die Medizin als recht fruchtbar. Die gute Erfahrung besteht laut Zimmermann aus Gelehrsamkeit, Beobachtung (dies war vor allen Dingen Hallers klinische Leitlinie) und «Genie». Mit letzterem vagen und problematischen Begriff, den er zusammen mit Lavater ausstreute, wurde Zimmermann ein Vorläufer der Sturmund-Drang-Literaten. Goethe, der laut eigener Angabe Zimmermanns Erfahrungsbuch sehr viel verdankte, hatte als Exstürmer und -dränger eigentlich wenig Grund, in «Dichtung und Wahrheit» über Zimmermanns Geniebegriff zu spotten.

Die eigentlich medizinischen Ausführungen Zimmermanns enthalten nichts über Experimente und erstaunlich wenig wirklich Beobachtetes. Es wird immer von den Beobachtungen «der Alten» gesprochen. Mit den damals neuesten Fortschritten der medizinischen Wissenschaft (z. B. Perkussion, wirksame SkorbutBehandlung etc.) ist der Autor nicht vertraut. Einzig auf dem Gebiet des Seelischen nimmt er manches vorweg wie «Organminderwertigkeit» oder «Refoulement ${ }^{5}$, läßt sich aber auch zu solchen psychogenetischen Exzessen hinreißen wie die Erklärung von Pockenepidemien, Pestepidemien und Ruhrepidemien als psychisch bedingt ${ }^{6}$. Diese psychogenetische Maskierung von Unkenntnis der Krankheitsursachen kann man allerdings durch die ganze Medizingeschichte bis auf den heutigen Tag verfolgen. Als andere uralte faule Ausrede für Unwissenheit 
diente früher die «Vererbung » von Krätze, Lepra, Kropf, Rachitis, Würmern etc.

Das Büchlein über den Nationalstolz ist wohl das lesbarste der Zimmermannschen Bücher. Es verdankt manches Montaigne und enthält bereits 1758 eine Prophezeiung der Revolution: «Wir leben in der Dämmerung einer großen Revolution, die hie und da einen Kopf kosten wird.»?

Das Lob der Einsamkeit von 1756, wie alle Zimmermannschen Bücher in zahlreichen sich stets erweiternden Auflagen erschienen, ist in eine Art präromantische Trauer gehüllt. Nicht umsonst beruft sich Zimmermann unablässig, sehr zum Ärger seines Lehrers Haller, auf Rousseau. Gleichzeitig ist aber Zimmermann natürlich um diese Zeit noch ein engagierter Aufklärer in der Art Montesquieus, und er erwählt darum Kirchenmänner, in diesem Fall Einsiedler und Mönche, als die Repräsentanten der «schlechten Einsamkeit». Er verschwendet eine entsetzliche Menge Platz an eine liebevolle Darstellung des Sexualverhaltens dieser Männer und Frauen und an eine Polemik mit einem Narren namens Obereit, der sie verteidigte. Manche seiner Ausführungen über die «Genies in zerrissenen Hosen ${ }^{8}$, wie er seine Objekte nennt, erinnern allerdings an unsere Gegenwart. Auch hier wieder gute psychiatrische Krankheitsbeschreibungen.

Das Buch über Friedrich II. oder den Großen ist wohl sein schwächstes. Es ist eine hauptsächlich gegen Mirabeau und Nikolai gerichtete Apologie des kürzlich Verstorbenen. Daß Zimmermann (und viele andere Deutsche, z. B. auch Goethe) damals Friedrich II. bewunderten, ist verständlich; daß er aber auch dessen Münzfälschungen und dessen Adelsprotektion darin einschließt und daß er solch traurige Figuren wie Friedrich Wilhelm I. und Friedrich Wilhelm II. mit derselben Bewunderung besingt, spricht nicht für ihn. Die Monarchenverehrung war jedoch nun einmal seine Spezialität geworden, und er erstreckte sie auch auf den Duke of York, auf Katharina II. oder die Große (die es schon ihren roten Nachfolgern vorgemacht hat, wie man «nützliche Idioten» im Westen als Propagandisten benutzt), Cäsar etc. Auch war Zimmermann vielleicht nicht gerade der rechte Mann, so heftig gegen die «Aufklärungsnarrheit» und seine alten Freunde wie Nikolai zu polemisieren.

Wie war nun eigentlich dieser Mann beschaffen, der so viel von sich reden machte und so viel von sich redete ? Sein alter Freund Tissot, diese anima candida, nennt ihn ein «enfant gâté». ${ }^{9}$ Und das war auch wohl Hallers Auffassung, der ihm gewogen blieb, obwohl Zimmermann sich doch, wie allerdings so mancher andere dankbare Schüler, ihm gegenüber später so wenig schön verhalten hatte ${ }^{10}$. Goethe, der ihm in «Dichtung und Wahrheit» 35 Jahre nach ihrer Freundschaft und 15 Jahre nach seinem Tode relativ viel Platz widmet ${ }^{11}$, gibt ohne weiteres $\mathrm{zu}$, daß er «in seiner Zeit sehr wirksam war, ebenso wie Tissot, Haller und 
Unzer ». ${ }^{12}$ Er erkennt auch dankbar an, daß er aus dem Erfahrungsbuch sehr viel gelernt habe. Daß Zimmermann die Frau von Stein auf Goethe aufmerksam machte, eh dieser nach Weimar kam, indem er sie vor seiner Faszination warnte ${ }^{13}$, erwähnt er nicht. Als Zimmermann 1775 Goethe in Frankfurt besuchte, fiel Goethe allerdings seine Eitelkeit unangenehm auf, auch seine Härte gegen seine Kinder. Auch mißfiel Goethe, daß er «auf alles losschlug», und er sagt weiter: «Seine leidenschaftliche Verbesserungswut konnte ich nicht mit ihm teilen». ${ }^{14}$ Er entschuldigt aber sehr viel mit Zimmermanns «Hypochondrie» (neurotischer Charakter).

Diese zwei zeitbedingten Züge, die Verbesserungswut und die Hypochondrie, erklären vielleicht Zimmermanns enormen Erfolg bei seinen Zeitgenossen mehr noch als seine unbezweifelbare Intelligenz und sein schriftstellerisches Talent. Es gab wahrscheinlich um diese Zeit nicht weniger Neurotiker als heute, und sie fühlten sich durch seine Schriften in gewisser Weise bestätigt und empfanden das als hilfreich. Die Verbesserungswut war damals geradezu epidemisch.

Zimmermann ist eine Inkarnation eines seit eh und je existierenden historischen Typus, der durchaus eine wichtige Rolle spielt, aber da er so ausschließlich zu seiner Zeit gehört, dazu tendiert, nach relativ kurzer Zeit total vergessen zu werden. An Beispielen für diesen fehlt es auch in unserer Zeit nicht. Wer, außer ein paar alten Leuten, erinnert sich heute zum Beispiel noch an die vor fünfzig Jahren so berühmten Oswald Spengler, Graf Hermann von Keyserling oder Emil Ludwig?

\section{Fußnoten}

1 s. Ackerknecht, E. H., und Buess, H., Kurze Geschichte der großen Schweizer Ärzte, Bern 1975, Kapitel «Aufklärung» S. 34-46.

${ }^{2}$ Bouvier, Aug., J.-G. Zimmermann, Genève 1925. Immer noch wertvoll S.A.D. Tissots letzte Schrift: Vie de M. Zimmermann, Lausanne 1797, und Bodenmann, Ed., J.G.Zimmermann: Sein Leben und Briefe an denselben, Hannover 1878.

3 Fischer-Homberger, E., Hypochondrie, Bern 1970.

4 Von der Ruhr unter dem Volke im Jahr 1765, Zürich 1767.

5 Von der Erfahrung in der Arzneykunst, S. 753 der Ausgabe von Zürich 1777; Bouvier l.c. S. 278.

6 Erfahrung 1777, S. 655.

7 Nationalstolz, Zürich 1758, S. 218.

${ }^{8}$ Einsamkeit, Ausg. Leipzig. 1785, Bd. II, S. 9.

9 Tissot 1797, S. 22.

10 Einsamkeit 1785, Bd. IV, S.168, 184; Bodenmann l.c. S. 275, $277 \mathrm{ff}$.

11 Goethe, J.W., Dichtung und Wahrheit, Büchergilde Gutenberg, Zürich 1938, S. 635 bis 639. 
12 ib. S. 268.

13 Briefe von Zimmermann an Frau von Stein in Goethes Gespräche, hrsg. von F. v. Biedermann, Insel-Verlag, Reutlingen 1957, S. 53, 57.

14 Goethe 1.c. S. 638, 639.

\section{Summary}

J. G. Zimmermann (Brugg 1728-Hanover 1795) studied in 1747-1751 in Goettingen under Haller, who also found him 3 jobs and a wife. Yet he did not make his contribution as a scientist, but as a fashionable philosopher. While city physician at Brugg he wrote 6 books. The best known are "On loneliness" and "On medical experience". As a court physician at Hanover (1768-1795) he wrote only a hagiography of Frederic the Great. Goethe, who knew him well, writes that in his time he was as influential as Tissot, Haller and Unzer.

Prof. Dr. med. Erwin H. Ackerknecht

Ottikerstraße 42

CH-8006 Zürich 